\title{
Study of Osmophilic Yeasts producing Invertase
}

\author{
By M. PAMELA SCARR AND D. ROSE \\ Tate and Lyle Ltd., Research Laboratories, Keston, Bromley, Kent, England
}

(Received 31 March 1966)

\section{SUMMARY}

Samples of sugar cane (Saccharum officinarum), cane juice, raw sugar and refinery samples were screened for osmophilic yeasts able to ferment sucrose. One of the isolates showed properties bordering on the original definition of an obligate osmophil. Therefore, diagnostic tests were adapted to fit ecological considerations. These modifications in technique were adopted for all isolates when it was found that more consistent results could thus be obtained. As a result of these tests relevant organisms were identified as Torulopsis apicola, T. globosa, T. lactis-condensi, T. bacillaris, Candida guilliermondii, Saccharomyces florentinus and a new species, $T$. kestoni.

\section{INTRODUCTION}

It has been known for some years (Scarr, 1951) that Saccharomyces rouxii and $S$. mellis, growing in impure concentrated sucrose solutions, will ferment any invert sugar present to form organic acids when the subsequent decrease in $\mathrm{pH}$ value allows a slow chemical inversion to follow. There was no detectable invertase production by normal diagnostic tests. Torulopsis species, growing more slowly, were also noted, but, under the conditions of test, did not appear to be biochemically active. Recently, however, an incident was reported of fermentation in a saturated sucrose solution containing no invert sugar and from it only torulopsis species were isolated. The following account describes the isolation and identification of osmophilic yeasts from this and other sources.

\section{METHODS}

Definition of concentration. Osmophilic yeasts grow in a highly selective medium. At very high concentrations of dissolved solids, the exact concentration is important and unless otherwise stated is defined here as ${ }^{\circ}$ Brix. This is the weight of dissolved solids as $\%$ of weight of solution determined at $20^{\circ}$.

Bacteria will grow, albeit slowly, in solutions up to $40^{\circ}$ Brix and yeasts and moulds up to $75-79^{\circ}$ Brix. For convenience, osmophilic organisms are defined here as those which can grow at concentrations over $65^{\circ} \mathrm{Brix}$, which in pure sucrose solutions are conditions near to saturation (saturated sucrose solution at $20^{\circ}=66.7{ }^{\circ} \mathrm{Brix}$ ).

Isolation, screening and storage. Plate cultures were made on osmophilic agar (Scarr, 1959) and colonies picked off into filter-sterilized $20 \%$ (w/v) sucrose yeastwater solution containing a trace of bromocresol green, in McCartney bottles with a Durham tube. These were incubated for a maximum of 3 weeks at $27^{\circ}$. The contents of bottles showing acid and gas formation were then transferred to $65^{\circ}$ Brix sucrose solutions containing 0.5\% Difco Bacto Peptone, 0.1\% $\mathrm{MgSO}_{4} .7 \mathrm{H}_{2} \mathrm{O}$ and $0.2 \%$ $\mathrm{KH}_{2} \mathrm{PO}_{4}$ (sterilized at $5 \mathrm{lb}$./in. ${ }^{2}$ for $10 \mathrm{~min}$.) and incubated at $27^{\circ}$ for a further 3 
weeks. Cultures still showing a positive result were then streaked on osmophilic agar and stored on slopes of this medium.

Isolates from cane, cane juice, air (entrapped on a membrane filter), raw sugar and intermediate refinery products were screened in this manner.

Diagnostic tests. Preliminary tests were made by the standard methods of Lodder \& Kreger van-Rij (1952) but using liquid auxanogram tests and growth on vitaminfree medium according to Wickerham (1951). The fermentation tests were incubated for 3 weeks at $27^{\circ}$.

Subsequently modifications were made in the fermentation and carbon assimilation techniques by using $10 \%(\mathrm{w} / \mathrm{v})$ of the appropriate sugar or carbon source, except for ethanol, inulin, succinic, lactic and citric acids which were tested at normal strength and salicin at $1 \%$ and in saturated solution. Assimilation of soluble starch was tested by the plate auxanogram.

Liquid assimilation media were prepared with Difco Yeast Nitrogen Base and carbon source at final strength, sterilized by filtration and dispensed aseptically as $5 \mathrm{ml}$. volumes into empty plugged sterile tubes. In the nitrogen assimilation test the Difco Yeast Carbon Base was re-inforced with extra glucose where necessary.

Inocula were taken from 72 -hr slopes on osmophilic agar (incubated at $30^{\circ}$ ), washed and centrifuged in sterile Ringer's solution; one drop of washed suspension was used per tube. We noticed no loss of viability after this treatment.

\section{RESULTS}

One hundred isolates were examined. After screening tests and the elimination of obvious duplicates, the number of cultures was ten. For comparative purposes, a culture of Saccharomyces rouxii, Boutroux (code Sa 24) isolated from a Mauritius raw sugar, was included in the examination. The ten species isolated fell into three genera which are considered separately.

\section{Cultures $T \% A, T 8, T 9, T 10, T 11$ and $T 12$}

Data for this series of isolates are summarized in Tables 1 and 2.

In Culture T7A (isolated from a refinery sample) $10 \%$ sugar broths only gave a fermentation pattern corresponding to Torulopsis apicola Hajsig (1958). From this aspect and its very poor growth on wort agar, it resembles the 'obligate osmophil' of Kroemer \& Krumbholtz (1931); but many osmophilic yeasts can be gradually accustomed to more standard conditions, so we do not consider this a difference of taxonomic significance. Assimilation of L-sorbose, xylose and adonitol were also positive at $10 \%$ concentration only.

Culture T 8 (isolated from raw, unrefined syrup) was a typical isolate of Torulopsis globosa (Olson \& Hammer); but here again, fermentation tests gave more consistent results at the higher concentration.

The biochemical reactions of Culture T9 (isolated from the air) at first indicated a connexion with Torulopsis dattila; but this was discounted as our isolate was a very much smaller organism than the authentic strain from the Centraal Bureau voor Schimmelcultures, Delft, Holland (CBS) and differed in growing without added vitamins, and growing strongly with ethanol as carbon source. It is smaller than $\boldsymbol{T}$. bacillaris and utilizes more carbon compounds in the full auxanogram. Any con- 
nexion with $T$. apicola can also be eliminated, since Culture T9 ferments glucose and sucrose more quickly, with the additional fermentation of raffinose; and again, it assimilates more compounds. We confirm the results of Dr W. Slooff (private communication), that the assimilation pattern resembles that of Candida guilliermondii, although more significant differences in fermentation become apparent when tested at $10 \%$ concentrations. Culture T 9 may be related to $C$. guilliermondii, but it must be ascribed to Torulopsis as it is asporogenous, forms no pseudomycelium on potato or corn-meal agar slide-cultures, and does not split arbutin. It is also a point of difference that we found the assimilation tests on salicin and cellobiose were only

Table 1. Morphological characteristics of osmophilic yeasts

\begin{tabular}{|c|c|c|c|c|}
\hline Code & $\begin{array}{l}\text { Growth in } \\
\text { wort broth }\end{array}$ & $\begin{array}{l}\text { Growth on } \\
\text { wort agar }\end{array}$ & $\begin{array}{l}\text { Pseudo- } \\
\text { mycelium }\end{array}$ & Ascospores \\
\hline $\mathbf{T 7}$ & $\begin{array}{l}(1-1 \cdot 5) \times(2-2.5) \mu^{*} \\
\text { sediment only }\end{array}$ & $\begin{array}{l}(1-1.5) \times(1.5-2.5) \mu \\
\text { growth poor, browns }\end{array}$ & Negative & Not formed \\
\hline T8 & $\begin{array}{l}(4-6 \cdot 5) \times(4.5-6.5) \mu \\
\text { sediment only }\end{array}$ & $\begin{array}{l}(4 \cdot 5-7 \cdot 5) \times(5-7 \cdot 5) \mu \\
\text { growth poor, cream }\end{array}$ & Negative & Not formed \\
\hline T9 & $\begin{array}{l}(1.5-2.5) \times(2-3.5) \mu \\
\text { sediment only }\end{array}$ & $\begin{array}{l}(0.75-1.5) \times(1.5-3) \mu \\
\text { browns, growth poor }\end{array}$ & Negative & Not formed \\
\hline T 10 & $\begin{array}{c}(1 \cdot 5-3) \times(2-5) \mu \\
\text { sediment only }\end{array}$ & $\begin{array}{l}(1 \cdot 5-3) \times(2 \cdot 5-5) \mu \\
\text { dark brown, growth } \\
\text { poor }\end{array}$ & Negative & Not formed \\
\hline T11 & $\begin{array}{l}(3 \cdot 5-5) \times(2 \cdot 5-5 \cdot 5) \mu \\
\text { sediment, thin ring } \\
\text { and faint islets }\end{array}$ & $\begin{array}{l}(2.7-4.5) \times(3.5-6.5) \mu \\
\text { white, creamy, margin } \\
\text { entire }\end{array}$ & Negative & Not formed \\
\hline T12 & $\begin{array}{l}(2 \cdot 5-5) \times(3 \cdot 5-5) \mu \\
\text { sediment only }\end{array}$ & $\begin{array}{l}(2 \cdot 5-4) \times(2 \cdot 5-5) \mu \\
\text { pale brown, growth } \\
\text { poor }\end{array}$ & Negative & Not formed \\
\hline $\mathbf{P 4 A}$ & $\begin{array}{l}(1 \cdot 5-4) \times(2-5) \mu \\
\text { sediment, ring and } \\
\text { islets }\end{array}$ & $\begin{array}{l}(1-4) \times(1 \cdot 5-4) \mu \\
\text { smooth, glistening, } \\
\text { creamy-white }\end{array}$ & $\begin{array}{l}\text { Blastodendrion } \\
\text { type }\end{array}$ & Not detected \\
\hline P4B & $\begin{array}{l}(1 \cdot 5-4) \times(2-5) \mu \\
\text { sediment, ring and } \\
\text { islets }\end{array}$ & $\begin{array}{l}(1-4) \times(1 \cdot 5-4) \mu \\
\text { smooth, glistening, } \\
\text { creamy-white }\end{array}$ & $\begin{array}{l}\text { Blastodendrion } \\
\text { type }\end{array}$ & Not detected \\
\hline $\mathrm{Sa24}$ & $\begin{array}{c}(2-4) \times(3-7 \cdot 5) \mu \\
\text { sediment only }\end{array}$ & $\begin{array}{l}(3-6) \times(3 \cdot 5-7 \cdot 5) \mu \\
\text { white-dirty-white, } \\
\text { viscous }\end{array}$ & Negative & $\begin{array}{l}\text { In newly isolated } \\
\text { cultures }\end{array}$ \\
\hline $\mathrm{Sa} 26$ & $\begin{array}{l}(3-5 \cdot 5) \times(5 \cdot 5-9) \mu \\
\text { sediment, thin ring } \\
\text { and islets }\end{array}$ & $\begin{array}{l}(2 \cdot 5-6) \times(4 \cdot 5-8 \cdot 5) \mu \\
\text { creamy-white, margin } \\
\text { crenated }\end{array}$ & $\begin{array}{l}\text { Long, slender } \\
\text { cells, sparse }\end{array}$ & Not detected \\
\hline
\end{tabular}

* Cell sizes of 3-day cultures, macroscopic appearance after 1 month at ambient temperature.

positive with inocula prepared by Wickerham's (1951) starvation method. With washed organisms as inocula (a necessity here, particularly with some of the 'obligate osmophils') the results were negative; $\beta$-glucosidase production is probably therefore very slight. With this combination of characteristics, we consider this to be a new species which we have named $T$. kestoni. A culture has been deposited at the Centraal Bureau voor Schimmelcultures. The Latin diagnosis is included at the end of this account.

Culture T10 (isolated from unrefined liquor) can be directly typed as Torulopsis lactis-condensi (Hammer). Culture T11 (also isolated from unrefined liquor) shows 
Table 2. Biochemical characteristics of Torulopsis spp.

\begin{tabular}{|c|c|c|c|c|c|c|c|c|c|c|c|c|}
\hline & \multicolumn{12}{|c|}{ Cultures } \\
\hline & \multicolumn{2}{|c|}{$\mathrm{Tr}$} & \multicolumn{2}{|c|}{ T8 } & \multicolumn{2}{|c|}{$\mathbf{T} \mathbf{9}$} & \multicolumn{2}{|c|}{ T10 } & \multicolumn{2}{|c|}{ T11 } & \multicolumn{2}{|c|}{ T12 } \\
\hline & $10 \%$ & $2 \%$ & $10 \%$ & $2 \%$ & $10 \%$ & $2 \%$ & $10 \%$ & $2 \%$ & $10 \%$ & $2 \%$ & $10 \%$ & $2 \%$ \\
\hline \multicolumn{13}{|c|}{ Fermentation } \\
\hline Glucose & + & - & + & $\mathbf{V}^{*}$ & + & + & + & + & + & + & + & + \\
\hline Sucrose & + & - & + & VW & + & VW & + & + & + & + & + & + \\
\hline Raffinose & - & - & + & VW & + & - & + & + & + & - & + & + \\
\hline Maltose & - & - & - & - & - & - & - & - & - & - & - & - \\
\hline Galactose & - & - & - & - & - & - & - & - & - & - & - & - \\
\hline Lactose & - & - & - & - & - & - & - & - & - & - & - & - \\
\hline Melibiose & - & - & - & - & - & - & - & - & - & - & - & - \\
\hline
\end{tabular}

Assimilation of carbon compounds

\begin{tabular}{|c|c|c|c|c|c|c|c|c|c|c|c|}
\hline Glucose & + & + & + & + & + & + & + & + & + & + & + \\
\hline Galactose & $\mathbf{W}$ & - & - & - & + & - & - & - & + & $\mathbf{W}$ & - \\
\hline Sucrose & + & + & + & + & + & + & + & + & + & + & + \\
\hline Maltose & - & - & + & + & + & - & - & - & + & $\mathbf{W}$ & - \\
\hline Lactose & - & - & - & - & - & - & - & - & - & - & - \\
\hline L-sorbose & + & - & + & + & + & + & - & - & + & + & - \\
\hline Cellobiose & - & - & - & - & + & - & - & - & - & + & - \\
\hline Trehalose & - & - & + & + & - & - & - & - & nt & + & + \\
\hline Melibiose & - & - & - & - & + & - & - & - & + & + & - \\
\hline Raffinose & + & + & + & + & + & + & + & + & + & + & + \\
\hline Melizitose & - & - & - & - & W & W & - & - & nt & - & - \\
\hline Inulin & . & - & . & $\mathbf{W}$ & . & - & . & - & + & - & + \\
\hline Starch & . & - & . & - & . & - & . & - & - & - & - \\
\hline Xylose & + & - & - & - & + & - & - & - & nt & - & - \\
\hline $\mathrm{L}$-arabinose & - & - & - & - & - & - & - & - & - & - & - \\
\hline D-arabinose & - & - & - & - & - & - & - & - & - & - & - \\
\hline D-ribose & + & $\mathbf{W}$ & - & - & + & + & - & - & nt & - & - \\
\hline L-rhamnose & - & - & - & - & - & - & - & - & - & - & - \\
\hline Ethanol & . & + & . & + & . & + & . & - & - & . & $\mathbf{W}$ \\
\hline Glycerol & + & + & + & + & + & + & - & - & + & + & - \\
\hline Erythritol & - & - & - & - & $\mathbf{W}$ & - & - & - & - & - & - \\
\hline Adonitol & + & - & + & + & + & - & - & - & nt & - & - \\
\hline Dulcitol & - & - & - & - & - & - & - & - & nt & - & - \\
\hline D-mannitol & + & + & + & + & + & + & - & - & + & + & + \\
\hline $\begin{array}{l}\text { D-sorbitol } \\
\alpha \text {-methyl }\end{array}$ & + & + & + & + & + & + & - & - & + & + & $\mathbf{W}$ \\
\hline glucoside & - & - & + & + & + & + & - & - & + & + & + \\
\hline Salicin & - & - & - & - & $\mathbf{W}$ & - & - & - & - & - & - \\
\hline Lactic acid & $\cdot$ & - & . & - & - & $\mathbf{W}$ & - & - & - & . & VW \\
\hline Succinic acid & $\cdot$ & - & . & - & . & + & . & - & nt & . & + \\
\hline Citric acid & $\cdot$ & - & . & - & - & + & . & - & + & . & $\mathbf{W}$ \\
\hline Inositol & - & - & - & - & - & - & - & - & - & - & - \\
\hline Arbutin & \multicolumn{2}{|c|}{-} & \multicolumn{2}{|c|}{-} & \multicolumn{2}{|c|}{ - } & \multicolumn{2}{|c|}{ - } & - & \multicolumn{2}{|c|}{-} \\
\hline $\begin{array}{l}\mathrm{KNO}_{3} \\
\text { Growth in } \\
\text { vitamin-free }\end{array}$ & \multicolumn{2}{|c|}{-} & \multicolumn{2}{|c|}{+} & \multicolumn{2}{|c|}{-} & \multicolumn{2}{|c|}{+} & + & \multicolumn{2}{|c|}{ - } \\
\hline medium & \multicolumn{2}{|c|}{$\mathbf{W}$} & \multicolumn{2}{|c|}{+} & \multicolumn{2}{|c|}{+} & \multicolumn{2}{|c|}{-} & + & \multicolumn{2}{|c|}{+} \\
\hline Growth at $37^{\circ}$ & \multicolumn{2}{|c|}{ - } & \multicolumn{2}{|c|}{-} & \multicolumn{2}{|c|}{+4} & \multicolumn{2}{|c|}{-} & - & \multicolumn{2}{|c|}{-} \\
\hline
\end{tabular}

$* \mathrm{~V}=$ variable; $\mathrm{W}=$ weak.

$\dagger$ In T 11, $10 \%$ solutions only were tested, except where the lower concentrations were used as in ethanol, starch, etc. nt $=$ not tested.

‡ Growth at $37^{\circ}$ positive on osmophilic agar only. 
Table 3. Biochemical characteristics of osmophilic yeasts

Cultures

$\overbrace{10 \%=2 \%}^{\text {P4A }} \overbrace{10 \%{ }_{2 \%}}^{\text {P4B }} \overbrace{10 \%}^{\text {Sa24 }} \overbrace{2 \%}^{\text {Sa26 }}$

Fermentation

Glucose

Sucrose

Raffinose

Maltose

Galactose

Lactose

Melibiose

Glucose

Galactose

Sucrose

Maltose

Lactose

L-sorbose

Cellobiose

Trehalose

Melibiose

Raffinose

Melizitose

Inulin

Starch

Xylose

L-arabinose

D-arabinose

D-ribose

L-rhamnose

Ethanol

Glycerol

Erythritol

Adonitol

Dulcitol

D-mannitol

D-sorbitol

$\alpha$-methyl glucoside

Salicin

Lactic acid

Citric acid

Succinic acid

Inositol

Arbutin

$\mathrm{KNO}_{3}$

Growth in vitamin-free medium

Growth at $37^{\circ}$

$\begin{array}{llllllll}+ & + & + & + & + & + & + & + \\ + & + & + & + & - & - & + & + \\ + & \mathbf{w} & + & \mathbf{w} & - & - & + & + \\ - & - & \mathbf{w} & - & + & - & + & + \\ + & + & + & + & - & - & + & + \\ - & - & - & - & - & - & - & - \\ - & - & - & - & - & - & + & +\end{array}$

Assimilation of carbon compounds

\begin{tabular}{|c|c|c|c|c|c|c|c|}
\hline $10 \%$ & $1 \%$ & $10 \%$ & $1 \%$ & $1 \%$ & $10 \%$ & $10 \%$ & $1 \%$ \\
\hline+ & + & + & + & + & + & + & + \\
\hline+ & + & + & + & $\mathbf{W}$ & - & + & + \\
\hline+ & + & + & + & - & - & + & + \\
\hline+ & + & + & + & + & VW & + & + \\
\hline - & - & - & - & - & - & - & - \\
\hline+ & + & + & + & - & - & - & - \\
\hline+ & + & + & + & - & - & - & - \\
\hline+ & + & + & + & - & - & + & + \\
\hline+ & + & - & - & - & - & + & + \\
\hline+ & + & + & + & - & - & + & $r$ \\
\hline+ & + & + & + & - & - & + & T \\
\hline - & + & . & + & - & - & • & - \\
\hline . & - & . & - & . & - & . & - \\
\hline+ & + & + & + & - & - & - & - \\
\hline+ & + & + & + & - & - & + & W \\
\hline- & - & + & + & - & - & - & - \\
\hline- & - & + & + & - & - & - & 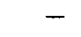 \\
\hline+ & + & + & + & - & - & - & - \\
\hline - & + & • & + & $\dot{x}$ & - & $\cdot$ & - \\
\hline+ & + & + & + & W & - & - & - \\
\hline+ & + & + & + & - & - & - & - \\
\hline- & - & + & + & - & - & - & - \\
\hline+ & + & + & + & - & - & - & - \\
\hline+ & + & + & + & - & - & - & - \\
\hline+ & + & + & + & - & - & - & - \\
\hline+ & + & + & + & $\mathbf{W}$ & W & + & + \\
\hline+ & + & + & + & - & - & - & - \\
\hline - & + & . & + & . & - & . & + \\
\hline . & + & . & + & . & $\mathbf{W}$ & . & - \\
\hline . & $t$ & • & + & . & - & • & - \\
\hline- & - & - & - & - & - & - & - \\
\hline & & \multicolumn{2}{|c|}{+} & \multicolumn{2}{|c|}{-} & \multicolumn{2}{|c|}{-} \\
\hline \multicolumn{2}{|c|}{+} & \multicolumn{2}{|c|}{-} & \multicolumn{2}{|c|}{-} & \multicolumn{2}{|c|}{-} \\
\hline & & \multicolumn{2}{|c|}{+} & \multirow{2}{*}{\multicolumn{2}{|c|}{$\overline{+}^{*}$}} & \multicolumn{2}{|c|}{+} \\
\hline & & \multicolumn{2}{|c|}{+} & & & & \\
\hline
\end{tabular}

* Growth at $37^{\circ}$ on osmophilic agar only. 
typical morphological characteristics of $T$. globosa (Olson \& Hammer) together with a strong reaction in the vitamin-free medium. It is unusual, however, in that it assimilates galactose and on the potato-agar slide cultures produces occasional organisms which show an abortive elongation similar to those in the authentic strain of $\boldsymbol{T}$. dattila. It may represent an intermediate form with species of the genus Candida, but these differences are not considered sufficiently decisive to justify the creation of a new variety.

Culture T12 (from a South American cane juice sample) was typed as Torulopsis bacillaris (Kroemer \& Krumbholtz). The negative reaction with $\mathrm{KNO}_{3}$ eliminated any relationship with $T$. lactis-condensi; the configuration of the organisms (singly and in pairs) in wort broth also eliminated the possibility of its being $T$. stellata which produces star-like clusters, or the variety $T$. stellata var. cambresier $i$ which splits arbutin.

\section{Cultures $P 4 A$ and $P 4 B$}

Culture $\mathbf{P} 4 \mathbf{A}$ was isolated from a West Indian sugar cane and Culture $\mathbf{P} 4 \mathrm{~B}$ from a South American cane juice. These two isolates were obviously identical and were identified as Candida guilliermondii (Cast.) Langeron et Guerra (Tables 1, 3). Formation of pseudomycelium on potato agar was observed after 7 days at $27^{\circ}$, but sporulation was not seen on V8 and Gorodkowa agar slants or carrot plugs even when mixed with a possible mating strain (Kreger van $R i j, 1964$ ). Culture $P 4 B$ is similar to a strain described by Kreger van Rij which assimilated melibiose. Of interest is the very high osmotic tolerance of these strains, a further indication of the cosmopolitan distribution of this species.

\section{Cultures Sa24 and Sa26}

Culture Sa 24 is a typical strain of Saccharomyces rouxii, but the fermentation of maltose was only definite in $10 \%$ solutions. This observation is similar to that of Santa Maria (1964) with Spanish cane molasses.

Culture Sa 26 was isolated from the air in a sugar factory and was identified as Saccharomyces florentinus, Castelli. Small amounts of mycelium only were seen on potato agar slide cultures after 7 days incubation. Sporulation was not observed on V 8 or Gorodkowa agar or carrot plugs after 12 weeks at $27^{\circ}$. To our knowledge, this is the first time that this organism has been found in an osmophilic habitat.

\section{DISCUSSION}

Barnett (1957) advocated the use of special biochemical tests for specific groups of yeasts, but the fat-splitting enzyme test used for the identification of Candida lipolytica shows that such an approach has been used for some time. The limitations of such tests are that often the media used are not sufficiently standard for biochemical use and merely form a medium for morphological observation, for example, Koji extract (Onishi, 1957) and honey agar (Lochead \& Heron, 1929); malt extract can be included in media of this type. On the other hand, increased sugar concentrations can be used in more chemically defined media.

In identifying these highly osmophilic yeasts either by the standard methods or by using an increased sugar concentration as here, consideration must be made of the nature of the original microhabitat; simply to record growth on an increased con- 
centration of sugar in an agar medium would appear to be insufficient (Scarr \& Rose, 1965). The practical assessment of the industrial importance of this group of osmophilic yeasts; either alone or with Saccharomyces rouxii and $S$. mellis would be in relation to the storage of raw sugar and other sucrose products for any length of time. This has yet to be determined.

Torulopsis kestoni sp.nov.

\section{Latin diagnosis}

In musto maltato cellulae subovoidae $(\mathbf{1} \cdot 5-2 \cdot 5) \times(2 \cdot 0-3 \cdot 5) \mu$, singulae, binae et glomerae. Post unem mensem, sedimentum.

In agara maltato cultura (post unem mensem $17^{\circ}$ ) fusca, tempore breve moritar. Pseudomycelium nullum.

Fermentatio ambis concentrationibus 2 et $10 \%$ glucosi et sacchari. Fermentatio raffinosi concentratione sola $10 \%$. In media minerale cum 1 et $10 \%$ glucoso, saccharoso, L-sorboso, raffinoso, D-riboso, glycerolo, D-mannitolo, D-sorbitolo et $\alpha$-methyl glucosido crescit. Cum $10 \%$ sola galactoso, maltoso, cellobioso, xyloso, adonitolo et salicino (exiguum) crescit. Cum $1 \%$ sola acido succinico, acido citrico et acido lactico (exiguum) crescit.

Arbutin non finditur. Formatio esterium nulla. Kalium nitricum non assimilantur. Non necessariae ad crescentium sunt vitaminae externae.

The authors wish to thank the Directors of Tate and Lyle Ltd., for permission to publish this paper and Miss W. Slooff of the Centraal-Bureau voor Schimmelcultures for her criticism and very valuable suggestions.

\section{REFERENCES}

Barnet, J. A. (1957). Some unsolved problems of yeast taxonomy. Antonie van Leeuwenhoek 23, 1.

HaJsig, M. (1958). Torulopsis apicola, nov. spec. New isolates from Bees. Antonie van Leeurvenhoek 24, 18.

Kreger-van Ris, N. J. W. (1964). Taxonomic Study of the Yeast Genera Endomycopsis, Pichia and Debaromyces. PhD. Thesis, University of Leiden, Netherlands.

Kroemer, K. \& Krumbholtz, G. (1931). Untersuchungen über osmophile Sprosspilze. I. Beiträge zur Kenntnis der Gärungsvorgänge und der Gärungserreger der Trockenbeerenauslesen. Arch. Mikrobiol. 2, 352.

Lochead, A. G. \& Heron, D. A. (1929). Microbiological studies of honey. Bull. Dep. Agnc. Dom. Can. No. 116 New Series.

Lodder, J. \& Kreger-van Ris, N. J. W. (1952). 'The Yeasts'. A Taxonomic Study. Amsterdam: North-Holland Publishing Co.

ONISHI, H. (1957). Studies on osmophilic yeasts. Part IIr. Classification of osmophilic soy and miso yeasts. Bull. agric. chem. Soc. Japan 21, 157.

SANTa Maria, J. (1964). Utilizacion de sacarosa y maltosa por levaduras. Boln. Inst. nac. Invest. agron. Madr. 50, 1.

SCARr, M. P. (1952). Osmophilic yeasts in raw beet and cane sugars and intermediate sugar-refining products. J. gen. Microbiol. 5, 704.

SCARr, M. P. (1959). Selective media used in the microbiological examination of sugar products. J. Sci. Food Agric. 10, 678.

SCARR, M. P. \& Rose, D. (1965). Assimilation and fermentation patterns of osmophilic yeasts in sugar broths at two concentrations. Nature, Lond. 207, p. 887.

Wickerham, L. J. (1951). Taxonomy of yeasts. Tech. Bull. U.S. Dep. Agric. No. 1029. 


\section{EXPLANATION OF PLATE}

Cultures mounted in glycerine jelly from a slope of osmophilic agar.

(a) Torulopsis kestoni ('T9). (b) T. bacillaris (T12). (c) Saccharomyces rouxii (Sa 24).

(d) Candida guilliermondii (P4B). 
Journal of General Microbiology, Vol. 45, No. 1

Plate 1
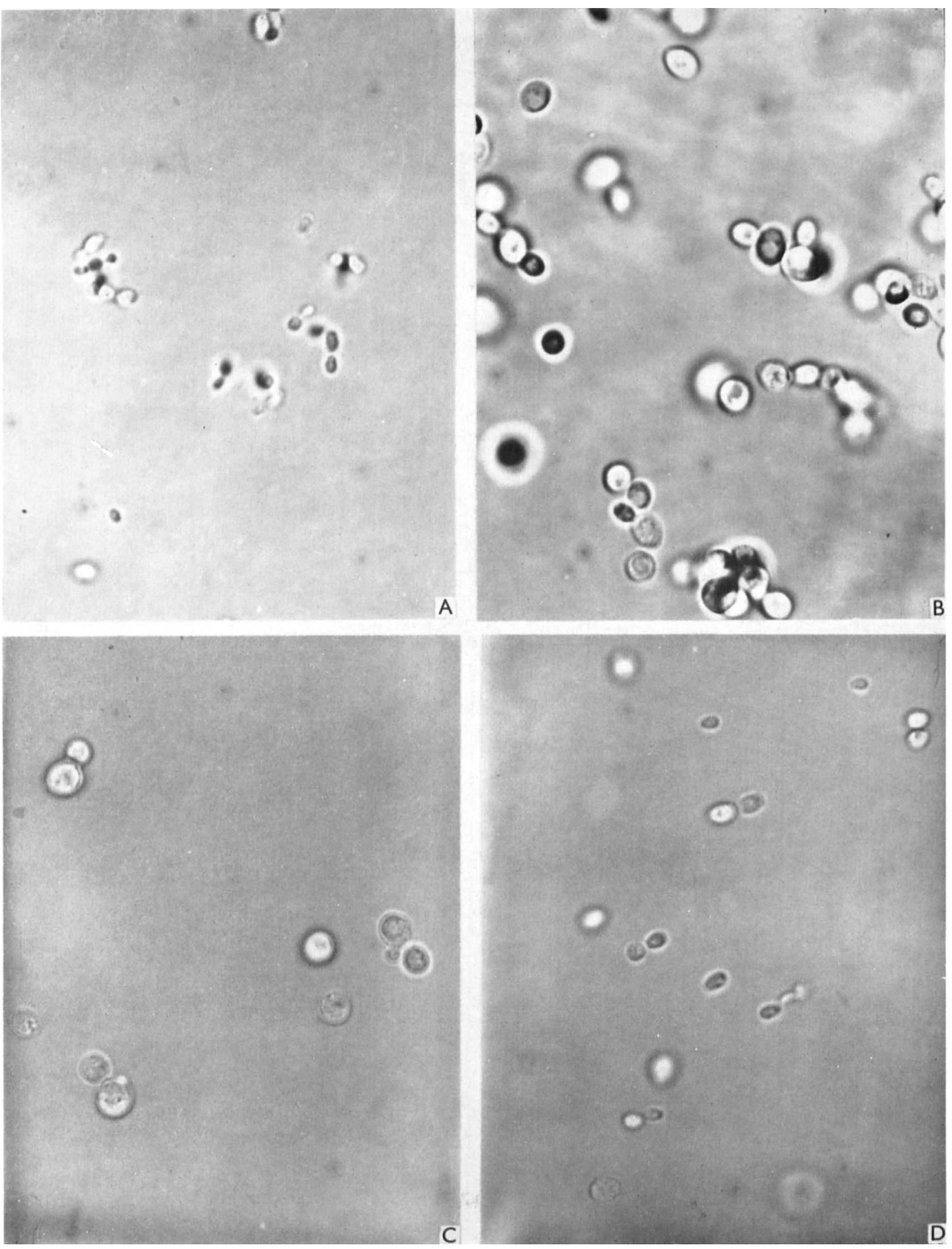

M. P. SCARR AND D. ROSE

(Facing p. 16) 